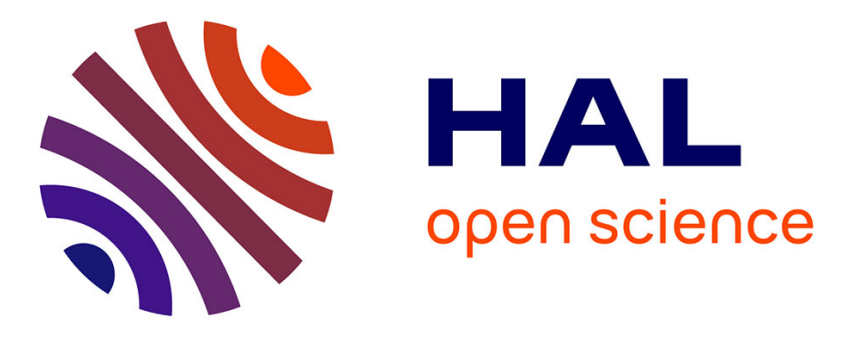

\title{
A Bio-Inspired Active Prostate Phantom for Adaptive Interventions
}

\author{
Stefan Escaida Navarro, Singh Dhaliwal, Mario Sanz Lopez, Sarah Wilby, \\ Antony Palmer, Wojciech Polak, Rochdi Merzouki, Christian Duriez
}

\section{- To cite this version:}

Stefan Escaida Navarro, Singh Dhaliwal, Mario Sanz Lopez, Sarah Wilby, Antony Palmer, et al.. A Bio-Inspired Active Prostate Phantom for Adaptive Interventions. IEEE Transactions on Medical Robotics and Bionics, In press, 10.1109/TMRB.2021.3123385 . hal-03374911

\author{
HAL Id: hal-03374911 \\ https://hal.inria.fr/hal-03374911
}

Submitted on 12 Oct 2021

HAL is a multi-disciplinary open access archive for the deposit and dissemination of scientific research documents, whether they are published or not. The documents may come from teaching and research institutions in France or abroad, or from public or private research centers.
L'archive ouverte pluridisciplinaire HAL, est destinée au dépôt et à la diffusion de documents scientifiques de niveau recherche, publiés ou non, émanant des établissements d'enseignement et de recherche français ou étrangers, des laboratoires publics ou privés.

\section{(ㅇ)(1) $\$$}

Distributed under a Creative Commons Attribution - NonCommerciall 4.0 International 


\title{
A Bio-Inspired Active Prostate Phantom for Adaptive Interventions
}

\author{
Stefan Escaida Navarro (D), Sepaldeep Singh Dhaliwal (D), Mario Sanz Lopez (D), Sarah Wilby, Antony \\ Palmer [D, Wojciech Polak, Rochdi Merzouki [D] and Christian Duriez (iD)
}

\begin{abstract}
In this paper, a novel concept for a phantom of the prostate is introduced. To study and improve automated, robotized medical interventions, reliable phantoms are essential. During brachytherapy and biopsy, the prostate will undergo displacements and changes in orientation due to the needle insertion. Therefore, there is a shift in the target regions for radioactive seed deposition or biopsy acquisition. These shifts need to be taken into account for precise dosimetry. Furthermore, the organ can undergo deformations due to edemas with the same shifting result. Therefore, in this paper we propose a bioinspired phantom (BIP) for the prostate that is equipped with sensors and coupled with a numerical simulation for estimating deformations due to external forces. We have also put in place a cavity inside the phantom where pressure can be applied to enable the emulation of prostate growth due to inflammation. The phantom is conceived in such a way that it will be MRIsafe. It can thus be deployed for the in-bore study of automated brachytherapy and biopsy. In our evaluation, we verify that the changes in pose of the phantom can be correctly estimated and that these motions are plausible from a clinical point of view.
\end{abstract}

\section{INTRODUCTION}

$\mathbf{P}$ ROSTATE cancer is one of the most common male cancers, particularly for men over 50 years of age. According to Cao et al. [1], among the age groups of 50-60, 65-74, and $75+$, incidence rates of $129.3,346.6,236.6$ per 100,000 men for all stages were respectively diagnosed between 2000-2017 $(95 \% \mathrm{CI})$. For the age group $<50$, the incidence is only 2.5 for the same period. Thus, there is a significant population, especially of age $>50$, that would profit from advances in the treatment of prostate cancer.

Brachytherapy is one of the most commonly opted treatment for prostate cancer. TRUS-guided (Transrectal Ultrasound) low-dose-rate (LDR) brachytherapy is routinely performed manually by physicians delivering up to $\approx 100$ radioactive seeds for treatment depending upon the required dosage and

Manuscript submitted on June 30, 2021. This work has been co-funded by the European regional development Fund under EU Interreg 2 Seas, Grant CoBra: 2S04-022. (Corresponding author: Stefan Escaida Navarro)

Stefan Escaida Navarro, Sepaldeep Singh Dhaliwal, Mario Sanz-Lopez and Rochdi Merzouki are with Centre de Recherche en Informatique, Signal et Automatique de Lille (CRIStAL) UMR CNRS 9189, University of Lille, 59650 Villeneuve d'Ascq, France (e-mail: stefan.escaidanavarro@inria.fr; sdhaliwal@univ-lille.fr; mario.sanz-lopez@univlille.fr; rochdi.merzouki@univ-lille.fr). Sarah Wilby, Wojciech Polak, and Antony Palmer are with Medical Physics Department,Portsmouth Hospitals NHS Trust, UK (e-mail: sarah.wilby@porthosp.nhs.uk; wojciech.polak@porthosp.nhs.uk; antony.palmer@porthosp.nhs.uk) Christian Duriez, INRIA Lille-Nord Europe, (e-mail:christian.duriez@inria.fr)

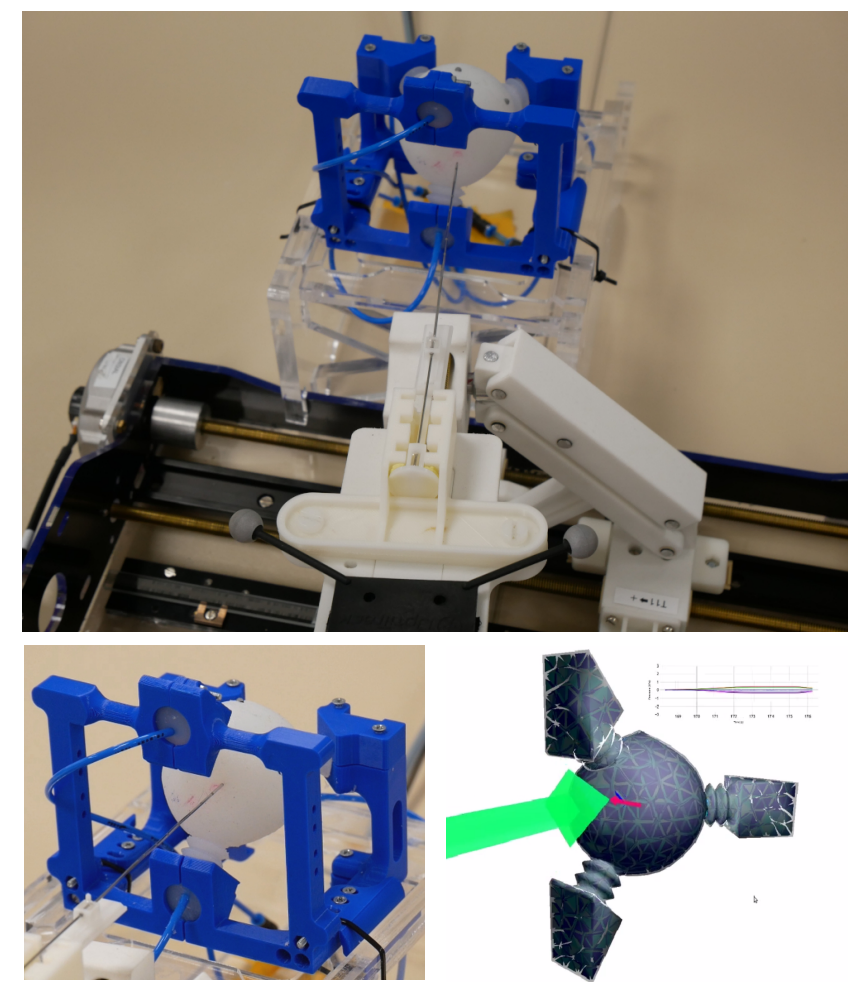

Fig. 1. In this work, we present a phantom with sensing and actuation capabilities that represents the prostate. Furthermore, we show the coupling of the phantom to the simulation framework SOFA. Top: The MRI-conditional robot developed in the CoBra-project carries a biopsy needle to perform a perforation test on the phantom. Bottom left: A view of the needle inserted into the phantom. Bottom right: A numerical FEM model of the phantom running in SOFA, where the interaction forces and the resulting deformations are estimated.

prostate volume 11 Fig. 2 illustrates the key aspects for the classical LDR brachytherapy procedure.

Currently, with the advent in high-performance imaging technologies for cancer detection, the focus with localized prostate cancer treatment and diagnosis is minimally invasive approaches/interventions. That is, targeting the lesion sites only while safeguarding the organs at risk (OARs) as well as the surrounding healthy tissues. In the last decade, as a solution to obtain the desired accuracy in reaching target lesion sites, many robotized solutions have been proposed and developed for prostate brachytherapy using ultrasound (US) image or magnetic resonance imaging (MRI) guidance [2].

${ }^{1}$ https://www.uhb.nhs.uk 
The robotic systems proposed for prostate biopsy and a few for brachytherapy using MRI guidance, have been summarized in [3]. Prostate phantoms can play an essential role in testing and validating the robotized solutions. In this paper, we propose such a phantom.

\section{A. Context and Contribution}

Our contribution takes place in the scope of the Cooperative Brachytherapy (CoBra) project. It proposes an integrated robotic solution for percutaneous intervention under realtime MRI. The team is developing a 5 Degree-of-freedom (Dof) MRI-conditional robot guide (see Fig. 1p, capable of performing interventions in-bor ${ }^{2}$ The interventions are the biopsy acquisition and brachytherapy itself. The CoBra concept emphasizes on adaptive dose delivery intraoperatively under real-time target tracking with the help of MRI in correlation with the planned dosimetry, compensating the target shift and edema due to needle puncture. Thus, the robot's control loop will be closed with MRI. Furthermore, an active omnidirectional steerable needle concept is proposed. The needle can deflect the distal tip to reach the new shifted target position. Moreover, by targeting the minimal invasive interventions, which reduce the tissue damage, the CoBra system is capable of performing oblique insertions with 2-3 perineum entry points while covering the whole prostate gland [4], [5].

These robots require an evaluation and validation on phantoms and cadavers to guarantee the desired accuracy of needle placement before any clinical testing. The prostate exhibits translational and rotational motions during needle interventions as well as edema caused by tissue damage. Therefore, in this paper, we propose an active, bio-inspired phantom (BIP), whose main goal is to enable the study and validation of automated interventions. One of its main features is the ability to track the deformation due to external forces, giving a foundation for studying algorithms that re-plan seed deposition based on the current deformation state of the organ. Furthermore, this phantom should also be of interest for medical training in general. The phantom is active in two senses: First, it is equipped with sensing and coupled to a simulation, thus yielding an online estimation of the forces and deformations on the phantom. Second, it can be inflated by air pressure in order to emulate the inflammation of the organ, which is also included in the modeling capabilities. Finally, we investigate the characteristics that make this BIP compatible with MRI. In Fig. 1, the CoBra-system of robot and phantom is shown. It is also shown how a needle insertion leads to an estimation of the force and deformation in the SOFA simulation $3 \mathrm{We}$ evaluate the accuracy of the state estimation of the BIP in the context of an automated needle insertion as well as in dynamic, manual interactions. The observed BIP shifts are compared to reference values reported in clinical studies.

The rest of the paper is structured as follows: In the next section, we give an overview of the literature pertaining the

${ }^{2}$ https://cobra-2seas.eu/2021/03/17/video-discover-the-cobra-latest-

advances/ 3 https://www.sofa-framework.org/

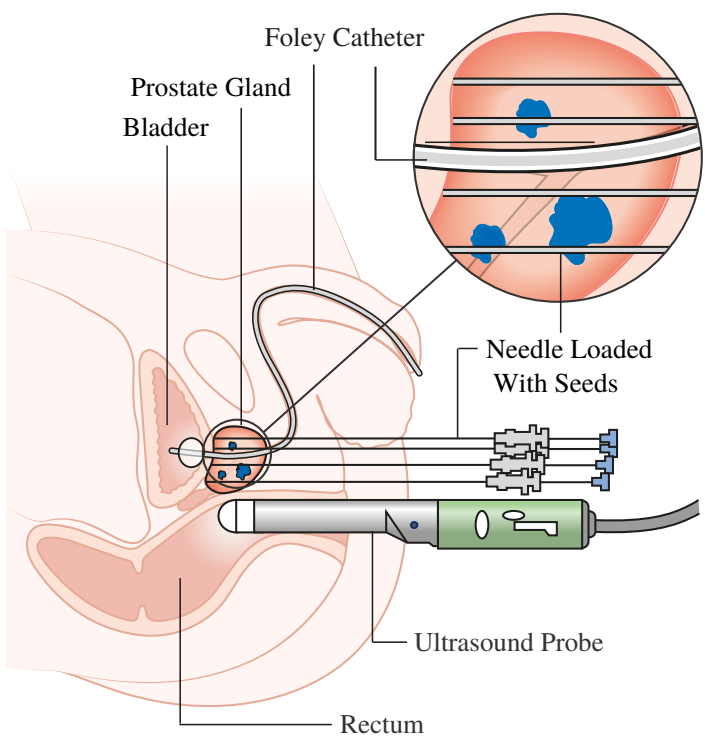

Fig. 2. Classical prostate brachytherapy (adapted from Wikimedia Commons [6]).

development of phantoms as well as sensing technologies similar to the ones employed here. In Sec. III] we provide the details of our BIP design. In Sec. [IV] we discuss the numerical modeling techniques that allow the estimation of forces and deformations on the BIP. Then, in Sec. $\mathrm{V}$, we discuss our experimental validation and provide results. Finally, in Sec.VI we provide concluding remarks and future perspectives for our line of work.

\section{RELATED WORK}

\section{A. Phantoms}

Phantoms are used to emulate the soft tissue behavior with similar mechanical properties to those of the real organs to test the needle intervention, study needle maneuvering, treatment planning and training of physicians. Smart phantoms can be helpful in mimicking the realistic behaviors for required evaluation and help in simulation for treatment planning. In the case of the prostate, the gland surrounds the urethra, and is in close proximity to the bladder and the rectum. It is an anatomically complex target for needle intervention as seen in Fig. 2

The phantoms used for the validation of the robots' needle placement are often static/passive in nature, developed inhouse or commercially available, e.g. CIRS Inc's (Model 53L/70L(Zerdine ${ }^{\circledR}$ gel)) or Yezitronix Group Inc 5 The passive phantoms are able to approximate the mechanical properties of prostate tissue, however, they are unable to estimate or represent the motions and edema effects.

In literature, it was found that the prostate phantoms are static and developed within laboratories by the research groups depending upon the objectives of research/projects such as dosimetric gels, ablation, or external beam radiation. Needle trajectory tracking or lesion targeting is mostly performed in

${ }^{4}$ https://www.cirsinc.com/products/

5 https://www.yezitronix.com/ 
static phantoms made of agar-agar, polyvinyl chloride (PVC) or commercialized phantoms, e. g. CIRS Inc. A recent review specifically of brachytherapy phantoms, highlighted the need to improve the tissue mimicking materials available for these [7]. Initial work related to anatomical prostate phantom was presented by Lindner et al. [8] and Betrouni et al. [9], who introduced a prostate anatomy and morphology for focal laser ablation application. Hungr et al. [10] developed a deformable and multimodal imaging compatible phantom. Choi et al. [11] presented phantom for transurethral resection, using biomimetic hydrogels with similar mechanical properties of prostate tissue. Niebuhr et al. [12] developed a pelvis phantom - ADAM (anthropomorphic, deformable and multimodal phantom), ADAM is capable of imagery outcomes with both MRI and CT, and suitable for long term use. Dynamic phantoms for liver to simulate tumor motion during breathing for realtime adaptive radiotherapy have been developed, e. g. [13] and [14].

In our knowledge only the pelvis phantom - ADAM [12] can be stated under active phantom category for prostate motion, the phantom generates motion of organs by inflating the urinary bladder with a syringe. The prostate attached to the bladder via a tube can move and twist. The phantom intends to reproduce the clinical treatment environment for adaptive radiotherapy. However, this phantom is not intended for needle intervention accounting only for organ-induced motions.

Considering the scenario of LDR brachytherapy for precise seed delivery and tracking target shift during needle intervention, an active prostate phantom is required to mimic the resulting prostate motions. The motivation behind the conception of bio-inspired prostate phantom (BIP) is to recreate a realistic scenario for adaptive prostate brachytherapy during robotized prostate intervention for the CoBra-project under the real-time MRI [15].

In this paper, we address the challenge of creating a phantom for the prostate that is suitable for studying robotized solutions for brachytherapy by taking into account the most important aspects, such as the organ displacement and change in orientation during the intervention. Our recent results regarding model-based sensing in Soft Robotics are the ideas upon which the proposed device is based. The BIP can be seen as an anatomical soft robot and in the presented approach we see the potential to represent further organs in a similar way in the future.

\section{B. Sensing for Soft Robotics}

Works close to our approach in terms of sensing are due to Truby et al. [16], Soter et al. [17], Tapia et al. [18] and Yang et al. [19]. Truby et al. show a complex sensorization of a soft finger, featuring a bending sensor, an inflation sensor and a contact sensor on the finger-tip. The bending measurement is shown to be independent of the inflation readings, meaning that it is possible, in principle, to estimate the bending radius independently of the pressure used for actuation, for instance, when the finger contacts an object. However, their work only shows results for regressions and does not deal with multiple interactions along different directions. Soter et al. propose a design in which cavities inside a soft pad are filled with a coloured liquid [17]. A display showing the level of the liquid in the cavity is used to monitor touch and bending interactions on two different devices. In terms of modeling, the sensor is identified at a single pressure point or bending along one direction through regression. Yang et al. [19] conceive a pneumatic sensor in the shape of a cuboid made out of silicone. They model the deformation of the wall carrying a weight similar to a beam. They find the beam deformation that best explains the pressure through the volume change, which results in the estimation of the force/weight. The same pneumatic sensor is then used to detect curvature by means of a geometric model (not a beam). However, this approach lacks an integration strategy, where shape and force can be estimated using the same model.

This work is a continuation of our recently shown approaches regarding model-based sensing for Soft Robotics using pneumatic sensors [20], [21]. In these works, we have demonstrated that a constraint-based approach using the FEM can be leveraged to model the behavior of pneumatic sensors that monitor pressure changes in cavities embedded in deformable structures. Therefore, using an optimization method, external, constraining forces on the model are found that best explain the observed changes in volumes and pressures. In this work, we show how these advances can be used to implement novel, smart devices for the medical technology domain.

\section{Concept, Design AND FAbrication of the BIP}

\section{A. Concept}

The main feature of the proposed BIP is its ability to detect external forces and thus the capability to represent and analyze interactions with it. Most notably, this includes the needle insertion during brachytherapy. However, we see this as a generic concept for anatomical soft robots, i. e. active, deformable devices that represent human anatomy and can be employed in validating automated interventions as well as in improved medical training.

\section{B. Design}

The main goal of the design of the BIP is to be able to reproduce the typical motions of the real organ that can be observed during interventions involving needle insertion. In Table I] we have summarized some findings from the literature about these movements [22], [23], [24]. Lagerburg et al. [22] use 3D US to retrieve the prostate rotational shift by referencing the position of the needle and the seeds at two points in time. Stone et al. [23] use $5 \mathrm{~mm}$ axial US images to determine translational shifts. Podder et al. [24] used a 6 DOF EM sensor for recovering the 3D translational data. According to these findings, it was determined that the BIP needs to reproduce translatory motions along the three spatial directions as well as the pitch and yaw rotations. Fig. 3 provides the reference axes for these movements. In the investigations shown, no movement in terms of rolling motion is reported. The BIP is, however, capable of reproducing these kinds of motions as well (see Sec. V). 


\begin{tabular}{|c|c|c|c|}
\hline Reference & {$[22]$} & {$[23]$} & {$[24$} \\
\hline $\begin{array}{c}\Delta \text { yaw (deg) } \\
\text { (coronal) }\end{array}$ & \pm 13 & $\mathrm{n} / \mathrm{a}$ & $\mathrm{n} / \mathrm{a}$ \\
\hline $\begin{array}{c}\Delta \text { pitch }(\mathrm{deg}) \\
\text { (sagittal) }\end{array}$ & \pm 8 & $\mathrm{n} / \mathrm{a}$ & $\mathrm{n} / \mathrm{a}$ \\
\hline$\Delta x(\mathrm{~mm})$ & $\mathrm{n} / \mathrm{a}$ & $3.6(1-5.5)$ & $1.4 \pm 0.6$ \\
\hline$\Delta y(\mathrm{~mm})$ & $\mathrm{n} / \mathrm{a}$ & $15(0-30)$ & $\begin{array}{c}12.6 \pm 4.5(17 \mathrm{G} 1.47 \mathrm{~mm}) \\
6.6 \pm 1.01(18 \mathrm{G} 1.27 \mathrm{~mm})\end{array}$ \\
\hline$\Delta z(\mathrm{~mm})$ & $\mathrm{n} / \mathrm{a}$ & $6.8(4.3-8.1)$ & $\mathrm{n} / \mathrm{a}$ \\
\hline
\end{tabular}

PROSTATE MOVEMENT DURING BRACHYTHERAPY ACCORDING TO LITERATURE. SEE FIG. 3 FOR A REFERENCE OF THE DIRECTION OF THE AXES

Furthermore, our BIP addresses the phenomenon of prostate volume growth due to edemas and inflammation caused by the repeated needle insertions, since literature suggests around $30 \%$ prostate volume increase during intraoperative interventions [25]. To this end, we use the space normally occupied by the urethra to put in place a cavity. Inflating this cavity will lead to a uniform growth of the prostate, much like a balloon, giving an approximation for the behavior due to inflammation. If dosimetry calculation is desired, a model including the gland's growth but with the urethra in its original location could be easily derived. The proposed method is suitable for any time scale and could cope easily with continuous growth during a brachytherapy session over 1.5-2 hours.

Beyond these clinical aspects, to us, modeling edemas is interesting, because it allows us to deploy numerical modeling techniques that combine the use of sensors and actuators to estimate a Soft Robotics device's (the BIP's) configuration. This also allows us to study further cases of adaptive needle navigation due to target shift or seed migration. Thus, even if the modeling of edema is coarse in the clinical sense (edemas can be localized rather than global), it can help us in improving the development of new types of phantoms, like the BIP. If not desired, removing edema emulation is trivially possible by not affecting the pressure of the urethra cavity.

We have thus designed the BIP such that it is capable of detecting a 3D vector of forces acting on a known, but arbitrary point $p_{\text {ext }}$ on its surface (see Fig. 3). Such a 3D force can induce the above-mentioned translations and rotations on the phantom, often in a coupled manner. For instance, an offcenter linear force on the phantom will push the phantom in the direction of that force, but also induce a torque and thus a pitch, yaw or even roll rotation.

The 3D forces are reconstructed by a model-based sensing approach that relies on pressure values as an input (see Sec. IV]. These pressure values are obtained from cavities inside the four arms that hold the BIP as shown in Figs. 3 and 4 The location and arrangement of these arms promotes the detection of the translatory and rotational movements: The two arms in the front are especially sensitive to displacements along the three spatial directions as well as to rotations about the $\mathrm{x}$-axis (pitch). The two arms in the back are especially sensitive to displacements along three spatial directions as well as to rotations about the z-axis (yaw). Overall, four sensors are used to estimate the three degrees of freedom of the linear forces, thus exhibiting a slight sensing redundancy.

The sensing arms also fulfill the role of representing the

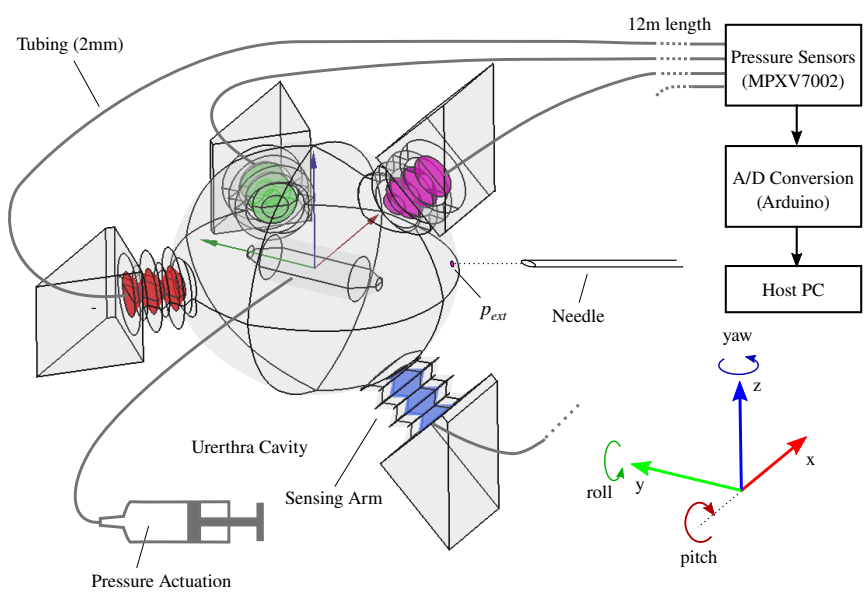

Fig. 3. Main elements of the BIP design: The layout of the cavities embedded in the sensing arms, the urethra cavity that can be inflated for reproducing inflammation, the needle and $p_{\text {ext }}$, the point on which it will interact with the BIP. The cavities are color coded to relate them to the plots in this paper and the attached video.
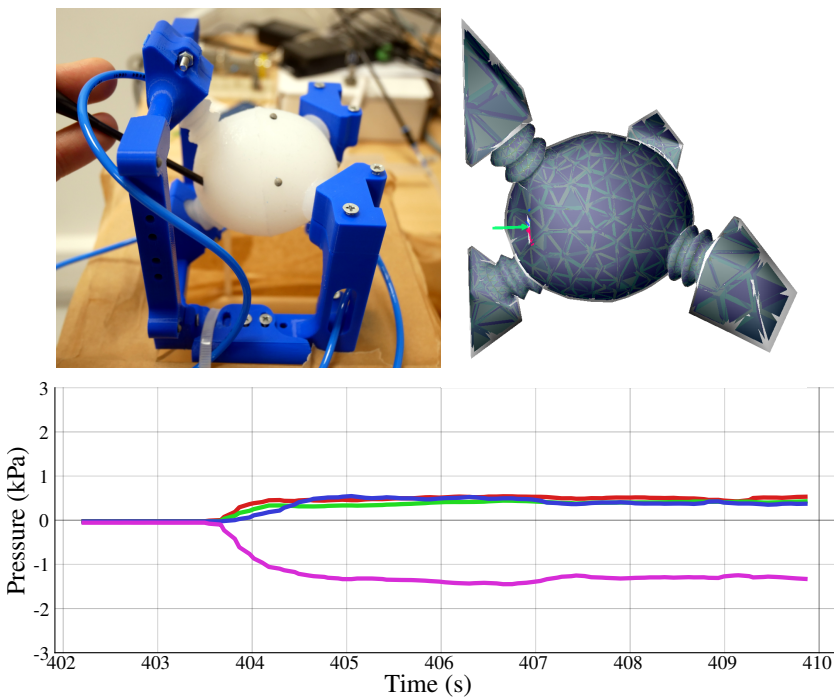

Fig. 4. Top: An example interaction with the BIP by hand (no perforation) with corresponding state estimation in SOFA. Bottom: The pressure signals recorded during this interaction. Due to the interaction, the four arms and the embedded cavities are deformed. Compression of the arms results in an increase in pressure and decrease in volume of the cavity and decompression the opposite.

elastic attachment of the prostate to the surrounding tissue and organs in a lumped manner. Thus, motion constraints due to the bladder, the rectum, ligaments, etc. are summarized by these arms. Their arrangement should approximate the motion behavior observed in clinical studies listed in Table I. The stiffness and arrangement (location, angle of separation) of the sensing arms will determine how much force is needed to change the configuration of the BIP in the spatial directions and orientations. Thus, it is in principle possible to emulate different conditions. By adding further elements, like the presence of a rigid US-probe, both to the BIP as well as to the simulation, a wider range of scenarios could be emulated.

To realize the design of the BIP, we relied on the free software Gmsh [26]. We used the Python API, which gives 
access to all of the software's functionality, to create the parametric geometry as well as the surface and volumetric meshes needed for visualization and simulation in SOFA. We have parameterized several aspects of the design, for instance the length of the sensing arms or their angle of separation. We were thus able to easily iterate over these parameters to find a suitable design.

\section{Fabrication}

For this study, the prostate phantom is made with silicone (Ecoflex ${ }^{\mathrm{TM}}$ 00-50, Smooth-On) ${ }^{6}$ According to the manufacturer's datasheet, the Shore hardness of 00-50 stands roughly at $83 \mathrm{kPa}(100 \%$ modulus) given that different mixture ratios lead to slightly different values. Carson et al. have studied the elastic modulus of whole-mount prostates, coming from prostatectomy and autopsy samples [27]. The samples include healthy prostates and prostates exhibiting pathologies. In this set, the overall mean elastic modulus is found to be $61.8 \mathrm{kPa} \pm 59.8 \mathrm{kPa}$. Thus, the elastic modulus of the selected material (Ecoflex 00-50) is higher than the average in this study, but within the range of observations. For future studies, we plan to work with materials having a wider range of elastic moduli. Its volume has been set to $100.41 \mathrm{~mL}$, which corresponds to a large prostate. Those choices, further versions and future improvements are discussed in Sec. VI

3D-printed molds were used to cast the silicone. The fabrication of the BIP took place in four different stages as: (1) With casting of sensing the arm core, that will result in the cavity, using silicone (Dragon Skin ${ }^{\mathrm{TM}}$ 30; Smooth-On), (2) Casting of prostate bulb and sensing arms using Ecoflex ${ }^{\mathrm{TM}}$ $00-50$, (3) Attachment of sensing arms to prostate bulb and pneumatic tubes to cavities using Sil-Poxy ${ }^{\mathrm{TM}}$, and (4) Mounting the assembly to 3D-printed frame.

The solid cores to template the cavities of the sensing arms were made of silicone as well to avoid damage or rupturing while extracting the core. Using more rigid materials, such as 3D-printed structures, we faced problems of tearing that rendered the sensing arms useless. To avoid the sticking of silicone to silicone, we applied a demolding agent. Fig. 5 shows the molds for BIP and the casted silicone prostate bulb with urethra cavity.

\section{Transducer Design and Performances}

The BIP has been designed to be integrated in a framework for MRI's in-bore environment. Thus, the whole system has been dimensioned to hold the electronics as far as possible from the MRI, to avoid EM interference. For this reason, in our tests, the cavities are attached to the electronics in a shielded box through $12 \mathrm{~m}$ tubing of $2 \mathrm{~mm}$ outer diameter $(1.2 \mathrm{~mm}$ inner diameter) that connects them to NXP MPXV7002 piezoresistive transducers [28]. Thus, the BIP itself has no electronic components near the MRI-apparatus and can be considered to be MRI-safe. The pressure sensors are connected to a first order low pass filter with a cut-off frequency of $600 \mathrm{~Hz}$ and sampled with an Arduino UNO (see also Fig. 37. In order

\footnotetext{
https://www.smooth-on.com/products/ecoflex-00-50/
}

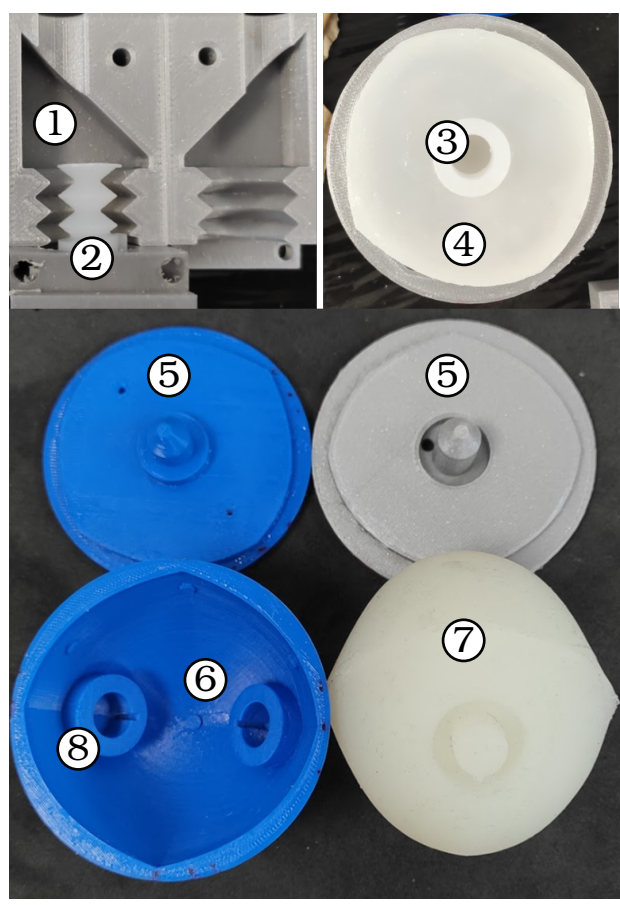

Fig. 5. BIP molds and parts: (1) sensing arm mold, (2) silicone core, (3) urethra cavity, (4) solid prostate, (5) cap for the molds, (6) sockets for placement of IR markers, (7) resulting bulb with no arms, (8) sockets for sensing-arm attachment.

to integrate correctly with the dimensions and environment of the prostate, the cavities have volumes of $486 \mathrm{~mm}^{3}$ for the horizontal sensing elements, and $514 \mathrm{~mm}^{3}$ for the vertical ones. Considering the extra volume introduced by the tubing, the overall volumes obtained are $14058 \mathrm{~mm}^{3}$ and $14086 \mathrm{~mm}^{3}$ respectively, which applying the ideal gases equation (5), and neglecting any thermal effects on the gases, any pressure detected after adding the tubing will be affected by a pressure loss $\left(\Delta P_{\text {loss }}\right)$ of 28.9 and 27.4 respectively. This factors are used to scale the calculated volumes.

To handle this decreased variability in pressures, the authors employed the mentioned MPXV7002 sensors, which are highly sensitive, featuring a measurement range of $\pm 2 \mathrm{kPa}$. Furthermore, the authors have taken advantage of oversampling techniques, with an oversampling ratio (OSR) of 100 samples per input. The Arduino UNO includes an Analog-toDigital converter (ADC) with a resolution $w$ of 10 bits, which making use of equations for oversampling ratio [29]:

$$
w+\log _{4}(O S R)
$$

gives a behavior equivalent to a resolution of approximately 13.32 bit of depth. The authors measured experimentally the maximum pressure values at the cavities during motion of the BIP, establishing $P_{\text {Activespan }}$ in a total range of $-7 \mathrm{kPa} /+10 \mathrm{kPa}$. Under these conditions, and with the MPXV7002 giving readings in the range of $P_{\text {MeasureSpan }}=$ $\pm 2 \mathrm{kPa}$, the digital span will be

$$
2^{w+\log _{4}(\text { OSR })} * \frac{P_{\text {ActiveSpan }}}{\Delta_{\text {PressureLoss }} * P_{\text {MeasureSpan }}}
$$


Giving 1505.9 and 1588.3 samples, or a resolution in the worst case scenario of $11.3 \mathrm{~Pa}$. The resulting subsystem is very sensitive and reliable, providing accurate readings with low ambient noise, and allowing for precise motion detection even on soft contact with the device.

Regarding the urethra cavity, it behaves in a slightly different manner, since it is not required to be held in a closed circuit, airtight environment, and it works at higher pressure values. Its cavity makes for a volume of $2078 \mathrm{~mm}^{3}$, it is actuated externally when inflammation is required, and it is connected to an NXP MPX4250D [30] pressure sensor.

\section{Numerical Modeling Techniques}

In this section we describe the numerical model that is implemented in our simulation framework SOFA $]^{7}$

\section{A. Online Finite Element Modeling (FEM)}

We use the FEM, which yields the internal elastic forces $\mathbb{F}(q)$, given that the nodes of the FEM mesh are at positions $q$ (see Fig. 11 right). In SOFA, we use a formulation that accounts for the geometric non-linearities of the deformation and the material is characterized by the Hooke's law (Young's modulus and Poisson's ratio). During each step $i$ of the simulation, a linearization of the internal forces is computed:

$$
\mathbb{F}\left(q_{i}\right) \approx \mathbb{F}\left(q_{i-1}\right)+K\left(q_{i-1}\right) d q,
$$

where $d q=q_{i}-q_{i-1}$ is the displacement of the nodes and $K=\frac{\partial \mathbb{F}\left(q_{i-1}\right)}{\partial q}$ is the tangential stiffness matrix for the current node positions $q$. To complete the picture, external forces are included:

$$
0=-K\left(q_{i-1}\right) d q+\mathbb{P}+\mathbb{F}\left(q_{i-1}\right)+H^{T} \lambda .
$$

$H^{T} \lambda$ is a vector that gathers boundary forces, such as contacts or external controlled inputs. The size of $\lambda$ is equal to the number of rows in $H$ and to the number of actuators (contact forces, cables, etc.). $\mathbb{P}$ represents known external forces, such as gravity. Then, (4) is solved under the assumption of static equilibrium, delivering a motion that is a succession of quasi static states. Please refer to [31] and [32] for a more in-depth discussion about the FEM formulation used here.

\section{B. External Forces Actuation}

In SOFA, it is possible to model external force actuators that simply apply forces on some point $p_{\text {ext }}$ on the model. In this work, this force actuation is used to represent the pushing or needle insertion on the BIP. The actuation magnitude is given by the variable $\lambda_{f}$, which can be distributed to a number of points $p_{i}$ on the FEM-mesh, as shown in Fig. 6 In this case, the forces are applied to a point on the surface of the object (magenta in Fig. 6) and then distributed on the FEM-nodes through a barycentric mapping (the circled orange points closest to $p_{\text {ext }}$ in Fig. 6). Since in this work we have knowledge of the desired the needle trajectory, we assume to have prior knowledge of the actuation point's location (see

https://www.sofa-framework.org/

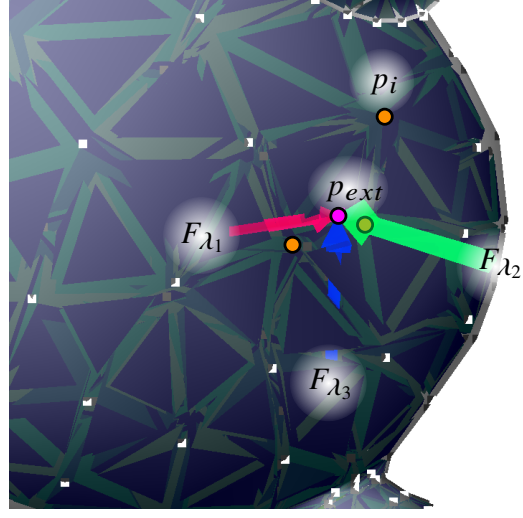

Fig. 6. Three forces along the world axis are applied to one point $p_{\text {ext }}$ (magenta). These forces are then distributed to the closest FEM-nodes $p_{i}$ (circled orange).

also Fig. 3). It should, however, be possible to equip the BIP with further sensing modalities to detect the location of the needle insertion interactively. In our previous work [21], we have addressed this sensor fusion challenge.

The resulting force $\vec{F}_{i}$ on each node $p_{i}$ is determined by the variable $\lambda_{f}$ and the 3D distribution $\vec{v}_{i}$ on nodes, i.e. $\vec{F}_{i}=\vec{v}_{i} \lambda_{f}$. The distribution is gathered in a row matrix $H_{f}=\left[\ldots, \vec{v}_{i}^{T}, \ldots\right]$, so that the expression $H_{f}^{T} \lambda_{f}$ represents the force actuation in (4).

\section{Pressure Actuation}

For simulation barymetric pressure, a variable $\lambda_{p}$ can be introduced. An internal cavity is represented by a triangulated surface mesh. Since pressure is force per area, the distribution of $\lambda_{p}$ on the nodes is calculated by taking into account the orientation and area of the triangles adjacent to each node, thus constructing the matrix $H$ in similar fashion than before. For further details, please refer to [32].

\section{Transforming Pressure Measurements to Volumes}

The force estimation method is based on volume information as inputs, not pressures. As we are using pressure sensors in the BIP, it is necessary to transform pressures to volumes. For now, we use the assumption that the pressure inside the sensing arms of the BIP does not significantly affect the shape of the cavity when it is being compressed by the external forces. In other words, we do not currently model the forces due to the pressure inside the pneumatic cavities, which in general could affect their shape and compliance. This is, however, an acceptable simplification for the setup we use, as the measured pressures are in the lower kPa-range (see Fig. 47. This way, we use the ideal gas law to transform from pressure readings to volumes (taking accounting the pressure loss factors described in Sec. III-D :

$$
P V=n R T,
$$

where $P$ is the pressure, $V$ the volume, $n$ the number of moles of gas, $R$ the ideal gas constant and $T$ the temperature. We assume furthermore that we have constant temperature, so that 
the right hand side of (5) is in fact constant. For the initial conditions we get:

$$
P_{0} V_{0}=n R T=C .
$$

Therefore, the relationship between pressure and volume for a measurement is:

$$
\Delta V=\frac{C}{\left(P_{0}+\Delta P\right)}-V_{0}
$$

\section{E. Finding Forces and Cable Displacement through Optimiza- tion}

Using inverse problem solving [32], we can optimize for the actuation force that best explains the volume changes inside the pneumatic sensors of the BIP (see Sec. IIII-D). The readings of the sensing arms of the BIP are converted according to (7) from the pressure measurement. Let $\Phi_{i}(q)$ be the function that maps the positions of the nodes of the FEM-mesh to the volume of a pneumatic cavity with index $i \in\{1,2,3,4\} . \Phi_{i}(q)$ can be calculated using the triangles of the mesh defining the cavity and is already implemented in SOFA.

The actuation forces due to pushing are given by $\Delta \lambda=$ $\left(\Delta \lambda_{1}, \Delta \lambda_{2}, \Delta \lambda_{3}\right)^{T}$. To find the relation between the change in applied forces and the change in the (simulated) volume $\Delta V_{i, s i m}$ of one pneumatic sensor we can write:

$$
\Delta V_{i, s i m}=\frac{\partial \Phi_{i}(q)}{\partial q} K^{-1} H^{T} \Delta \lambda
$$

Therefore, first the changes of forces are mapped to the corresponding nodes through $H^{T}$, as discussed in Sections IV-B and IV-C. The tangential compliance matrix $K^{-1}$ transforms these forces to (FEM-)node-displacements, which finally can be mapped to changes in volume through the derivative of $\Phi$ with respect to $q$. For conciseness, we rewrite $\frac{\partial \Phi_{i}(q)}{\partial q} K^{-1} H^{T}$ as $W_{i, v a}$, which is the matrix that directly maps changes in actuation force to changes in volume. Now, we can formulate the optimization problem. We want to minimize the difference between the simulated volumes $\Delta V_{\text {sim }}=\left(W_{1, v a} \Delta \lambda, \ldots, W_{N, v a} \Delta \lambda\right)^{T}=W_{v a} \Delta \lambda$ and the real changes in volume $\Delta V_{\text {real }}=\left(\Delta V_{1}, \ldots, \Delta V_{N}\right)^{T}$ :

$$
\begin{gathered}
\Delta \lambda_{\text {out }}=\underset{\Delta \lambda}{\arg \min }\left\|W_{v a} \Delta \lambda-\Delta V_{\text {real }}\right\|^{2}, \\
\text { s.t. } \Delta \lambda_{\text {max }} \geq \Delta \lambda \geq 0 \text { and } \\
\Delta \lambda_{P}=\Delta P_{\text {input }} .
\end{gathered}
$$

Finally, we write out the squared expression. There, we ignore the constant term corresponding to $\Delta V_{\text {real }}^{T} \Delta V_{\text {real }}$ for the optimization:

$$
\Delta \lambda_{\text {out }}=\underset{\Delta \lambda}{\arg \min }\left(\Delta \lambda^{T} W_{v a}^{T} W_{v a} \Delta \lambda-2 \Delta \lambda^{T} W_{v a}^{T} \Delta V_{\text {real }}\right) .
$$

This quadratic program $(\mathrm{QP})$ is solved using a $\mathrm{QP}$-solver with the method presented in [32].

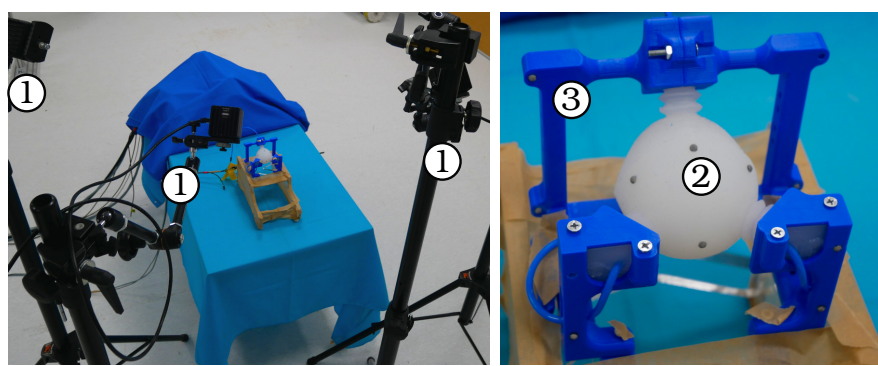

Fig. 7. The setup used to track the pose of the BIP using Optitrack cameras: (1) Optitrack ${ }^{\mathrm{TM}}$ Prime 13 cameras, (2) IR markers on the BIP, (3) Reference IR markers on the frame.

\section{Evaluation}

To validate the accuracy of the Bio-inspired phantom's $6 \mathrm{DoF}$ motion as reconstructed by the simulation, a tracking setup was put in place, as shown in Fig. 7. The tracking device consists of a set of 5 Optitrack $^{\mathrm{TM}}$ Prime 13 cameras run by Motive ${ }^{\mathrm{TM}}$. This camera set gives us absolute coordinates of the tracked points, thus providing a ground truth source with high precision. We registered the tracking data to the simulation with the help of referencing markers, with a precision determined by Motive ${ }^{\mathrm{TM}}$ of $\pm 0.19 \mathrm{~mm}$. Then, Motive ${ }^{\mathrm{TM}}$ streams the tracking data using the VRPN protocol, which is recovered by the vrpn_client_ros Packet of ROS. A Python package links the topics related to the received rigid bodies, adapts and filters the data if needed to inject them into the simulation. Within SOFA, the frames are overlaid and logged to files, which are the sources of the plots in Figs. 8,9 and 10 . There is a latency between the tracking and the simulation due to the processing of the numerical simulation as well as the communication pipeline. The latency adjustment needed to make the plots match is $35 \mathrm{~ms}$. However, the latency between the real event and the simulation result is probably around $100 \mathrm{~ms}$. We have adjusted for the latency in the plots for the sake of clarity/simplicity in the representation.

\section{A. Needle Insertion by the MRI-Conditional Robot}

In this first experiment, we used the setup of the MRIconditional robot (see Fig. 1) to conduct a needle insertion within the phantom. The needle is a $18 \mathrm{G}(1.27 \mathrm{~mm}$ diameter $)$ biopsy needle. This experiment is also documented in the attached video. Fig. 8 shows the output of the SOFA-model compared to the data of OptiTrack. The estimated data is shown as dashed lines, whereas the OptiTrack data is shown as solid lines. Overall, the estimated values follow the ground truth data quite satisfactorily. However, there is some bias, most noticeable in the $y$-translation, which is overestimated up to about $1 \mathrm{~mm}$. There is some noticeable angular displacement in the pitch-angle (here OptiTrack measurements are noisy) and the yaw-angle, which is accurately reproduced. Both, the model and OptiTrack, give a small output for the roll-angle, but the noise level of the data allows no comparison. We think this experiment is encouraging for the use of the BIP for studying and evaluating automated interventions. 

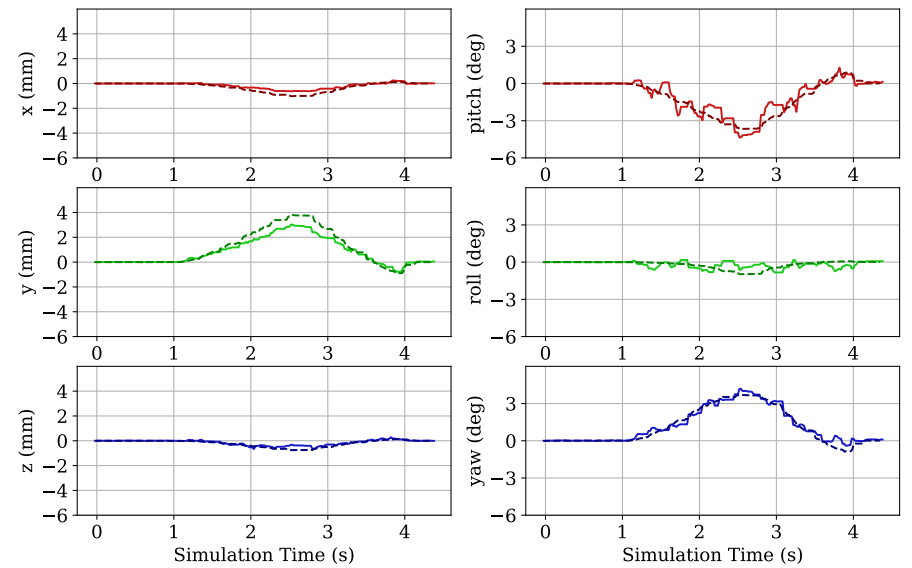

Fig. 8. Plot of the 6D-motion of the BIP during the needle insertion executed by the robot. Dashed lines are the estimated values, continuous lines are the OptiTrack ground-truth.
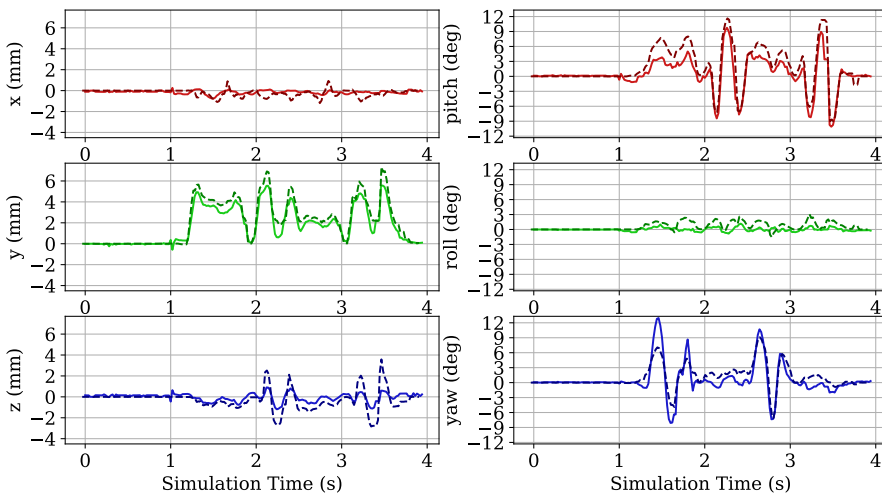

Fig. 9. Plot of the 6D-motion of the BIP during the manual interaction, on-center. Dashed lines are the estimated values, continuous lines are the OptiTrack ground-truth.
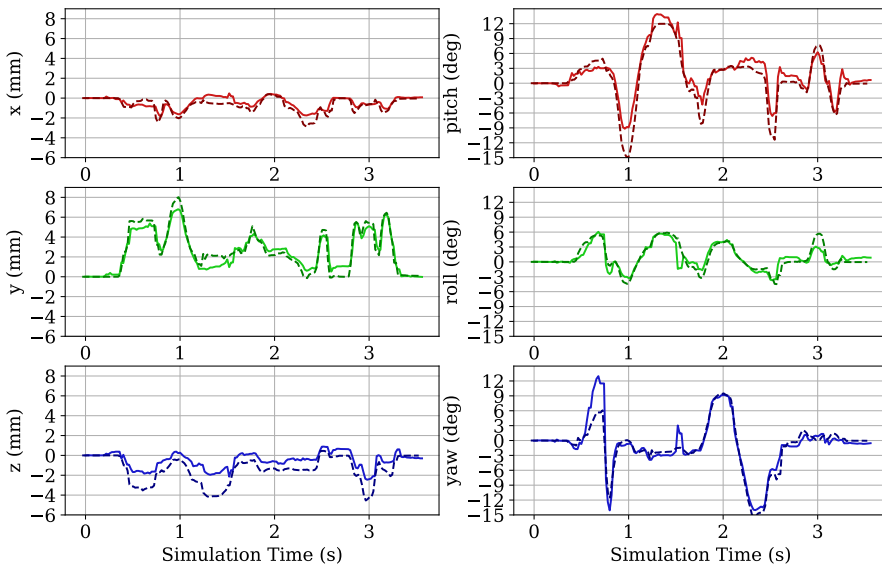

Fig. 10. Plot of the 6D-motion of the BIP during the manual interaction, off-center. Dashed lines are the estimated values, continuous lines are the OptiTrack ground-truth.

\section{B. Interactions by Hand}

To test the interaction capabilities with the phantom in a more dynamic scenario, we recorded data of two sequences executed by hand with a tool that does not penetrate the bulb (compare to Fig. 4 and the attached video). In the first sequence, we pushed directly in the front of the phantom, oncenter (results shown in Fig. 99. During the second sequence, the interaction point is diagonally off-center from the front by $10 \mathrm{~mm}$, much like in Fig. 11 (results shown in Fig. 10). In both cases, the forces and displacements were of higher magnitude compared to the experiment with the robot.

The results indicate that the $6 \mathrm{DoF}$ closely follow the trend of the ground-truth. However, SOFA will sometimes overestimate the deflection in some degrees of freedom, for instance the $y$ translation or the pitch-angle in Fig. 9 Conversely, deflection can also be underestimated, like the yaw-angle in the same case. In the on-center case, there's minimal change in the roll angle, but the off-center case shows that the roll angle can also have significant deflection, which is correctly captured by the model. Overall, the estimation of the $6 \mathrm{DoF}$ deflection is satisfactory in these cases and a promising base for future iterations of this type of devices.

Compared to the clinical data that we summarized in Table I the results from the experiments show general agreement. That is, translations are largest along the direction of insertion of the needle. An example of strong agreement would be the result of $y$-displacement observed when using $18 \mathrm{G}$ needle results as reported by [24]. The value of $6.6 \pm 1.01(18 \mathrm{G} 1.27 \mathrm{~mm})$, is quite similar to the maximal $y$ displacements of $\approx 6 \mathrm{~mm}$ (Fig. 9) and of $\approx 8 \mathrm{~mm}$ (Fig. 10) respectively. The translations along the $x$-axis in our experiments are also similar to the clinical reference data, whereas the $z$-translation is somewhat smaller to the data provided in Table I Overall, we think that the BIP displays plausible motions when comparing to that of clinical data. A next step for validating this aspect would be get feedback from experienced urologists in a user study. In this way, the BIP's design could be adapted to match the experts' expectations.

\section{Inflammation}

In Fig. 11, an example for the effect of the inflation of the urethra cavity is shown. A marker that is located next to the cavity is deflected by approximately $2 \mathrm{~mm}$. This can serve to emulate the shifting of target regions due to inflammation. Capturing the movement of the BIP due to external forces is still possible while the urethra cavity is being inflated. Please see the attached video for an example of this behavior.

\section{Towards a Patient-Specific, MRI-Safe Phantom}

At the current stage, the BIP can be deployed in a MRI-safe manner, mainly due to the possibility of keeping the sensing electronics far away from the MRI-apparatus (see Sec. III-D). The BIP presented here is of a uniform material, which is an advantage for the modeling aspect. However, this implies that it is missing features such as a prostate membrane or tumor regions, i.e. a multi-material composition. Furthermore, the 

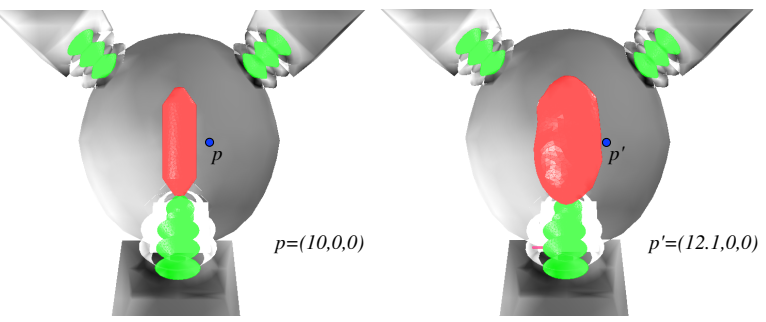

Fig. 11. The inflation of the urethra cavity causes a marker to be be deflected. This can be used to emulate the shifting of regions of interest in the real organ due to inflammation.

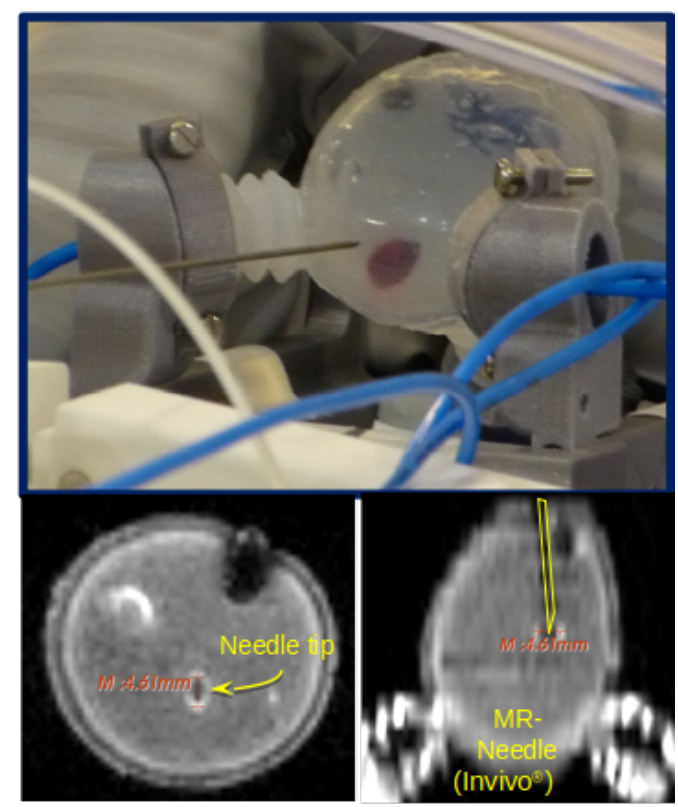

Fig. 12. Top: A new iteration of the BIP, featuring a transparent, harder shell and a soft Silicone Gel infill. Furthermore, lesion regions are also embedded. Bottom: MR-imaging results for the needle insertion procedure, displaying all the desired features, i.e. shell, infill, target and lesion regions as well as the needle.

materials that are suitable for the BIP-bulb might not be the best choice for the sensing arms, so a more advanced and realistic solution can easily consist of 3 or more different materials.

Currently, we are developing new iterations of the BIP and we have achieved in fabricating a version that has a shell of silicone of $1.5 \mathrm{~mm}$-thickness (Sorta Clear ${ }^{\mathrm{TM}}$ A12) and an infill with EcoFlex ${ }^{\mathrm{TM}} \mathrm{Gel}$. The sensing arms are made of EcoFlex ${ }^{\mathrm{TM}}$ 00-50. This version furthermore features lesion regions. In preliminary tests, we have performed needle insertion tests with the robot under MRI. Some results for the MRI are shown in Fig. 12. All the regions and features can be clearly identified, giving us confidence in this fabrication approach.

To achieve a fully functional BIP, we need to improve our modeling techniques, especially to account for the multimaterial aspect of membranes, infill, lesions, etc. Furthermore, we aim at creating patient specific phantoms. The geometry of the BIP will thus in the future be based on the anatomical data of the patient. With this, we could achieve a fully functional, patient specific, MRI-safe phantom for the prostate.

\section{SUMmARY AND CONCLUSIONS}

In this paper, we have shown the development of a bioinspired phantom (BIP) for the prostate that is capable of detecting external forces using a model-based sensing approach. The goal of this development is to improve and validate automated brachytherapy interventions. In this intervention, one of the main challenges is the movement of the organ induced by the needle insertion. To correctly place the radioactive seeds, the shifting of the organ needs to be taken into account. In our evaluation, we have shown that the BIP is able to estimate the displacements and changes in orientation. We have discussed that the shifts are plausible when compared to clinical data. Furthermore, it can, to some degree, emulate the effect of edemas and inflammation. This aspects are particularly important for the CoBra porject, as there is no other phantom capable of emulating any sort of seed migration problem.

Although we are optimistic about the obtained results, there are some discrepancies remaining between the estimated and real pose of the phantom. By further improving the design and modeling techniques, we think we can reduce this gap. We are also eager to look into more elaborate designs of the phantom, that are patient specific, i.e. based on patient anatomical data, and that also feature a multi-material composition. The advantage of such an approach is increased realism. Such a design can more correctly emulate the behavior of the needle insertion with a phase of puncturing the bulb and a phase of advancement within it. In general, we think that the proposed concept can serve to create active phantoms for other organs as well, which is an exciting opportunity to us. This particular phantom might also be useful for HDR prostate brachytherapy and cryotherapy.

To continue validating the proposed BIP, studies with actual seed deposition should be carried out. Here, we would like to study the impact of an adaptive, robotized intervention, featuring the steerable needle. Furthermore, we would like to get a validation from clinical experts in a user study to continue improving the design.

\section{ACKNOWLEDGMENT}

This work has been co-funded by the European regional development Fund under EU Interreg 2 Seas, Grant 2S04-022.

\section{REFERENCES}

[1] Y. Cao, W. Zhang, Y. Li, J. Fu, H. Li, X. Li, X. Gao, K. Zhang, and S. Liu, "Rates and trends in stage-specific prostate cancer incidence by age and race/ethnicity, 2000-2017," The Prostate, 2021.

[2] T. K. Podder, L. Beaulieu, B. Caldwell, R. A. Cormack, J. B. Crass, A. P. Dicker et al., "AAPM and GEC-ESTRO guidelines for image-guided robotic brachytherapy: Report of Task Group 192," Medical Physics, vol. 41, no. 10,2014

[3] S. S. Dhaliwal, T. Chettibi, S. Wilby, W. Polak, A. L. Palmer, N. Reynaert, and R. Merzouki, "Review of Clinical and Technological Consideration for MRI-guided Robotic Prostate Brachytherapy," IEEE Transactions on Medical Robotics and Bionics, vol. 3, no. 3, Jul. 2021.

[4] S. S. Dhaliwal, S. Wilby, S. Firouzy, K. B. Boni, M. de Vries, S. E. Navarro, A. Belarouci et al., "CoBra robot for localized cancer treatment and diagnosis under real-time MRI," AUTOMED 2021, June 2021. [Online]. Available: http://doi.org/10.5281/zenodo.4923036 
[5] S. S. Dhaliwal, S. Wilby, M. De Vries, K. Brou Boni, S. Firouzy, S. Escaida Navarro et al., "CoBra robot for localized cancer treatment and diagnosis under real-time MRI guidance," The Hamlyn Symposium on Medical Robotics Imperial College London, Jun. 2021, poster. [Online]. Available: https://hal.archives-ouvertes.fr/hal-03321170

[6] "Wikimedia commons," https://commons.wikimedia.org/wiki/File: Diagram_showing_how_you_have_high_dose_brachytherapy_for prostate_cancer_CRUK_419_pl.png. (Accessed on 08/31/2021).

[7] S. Wilby, A. Palmer, W. Polak, and A. Bucchi, "A review of brachytherapy physical phantoms developed over the last 20 years: clinical purpose and future requirements," Journal of Contemporary Brachytherapy, vol. 13, pp. $101-115,2021$

[8] U. Lindner, N. Lawrentschuk, R. Weersink, O. Raz, E. Hlasny, M. Sussman, S. Davidson, M. Gertner, and J. Trachtenberg, "Construction and Evaluation of an Anatomically Correct Multi-Image Modality Compatible Phantom for Prostate Cancer Focal Ablation," The Journal of Urology, vol. 184 1, pp. 352-7, 2010.

[9] N. Betrouni, P. Nevoux, B. Leroux, P. Colin, P. Puech, and S. Mordon, "An anatomically realistic and adaptable prostate phantom for laser thermotherapy treatment planning." Medical Physics, vol. 40 2, p. 022701, 2013

[10] N. Hungr, J. Long, V. Beix, and J. Troccaz, "A realistic deformable prostate phantom for multimodal imaging and needle-insertion procedures," Medical Physics, vol. 39 4, pp. 2031-41, 2012.

[11] E. Choi, F. Adams, S. Palagi, A. Gengenbacher, D. Schlager, P. Müller, C. Gratzke, A. Miernik, P. Fischer, and T. Qiu, "A High-Fidelity Phantom for the Simulation and Quantitative Evaluation of Transurethral Resection of the Prostate," Annals of Biomedical Engineering, vol. 48, pp. $437-446,2019$.

[12] N. Niebuhr, W. Johnen, G. Echner, A. Runz, M. Bach, M. Stoll, K. Giske, S. Greilich, and A. Pfaffenberger, "The ADAM-pelvis phantom-an anthropomorphic, deformable and multimodal phantom for MRgRT," Physics in Medicine \& Biology, vol. 64 4, p. 04NT05, 2019.

[13] A. G. de Merxem, V. Lechien, T. Thibault, D. Dasnoy, and B. Macq, "Design and implementation of a MRI compatible and dynamic phantom simulating the motion of a tumor in the liver under the breathing cycle," in Symposium on Medical Information Processing and Analysis, 2017.

[14] S. Ehrbar, A. Jöhl, M. Kuehni, M. Meboldt, E. O. Elsen, C. Tanner, O. Goksel, S. Kloeck, J. Unkelbach, M. Guckenberger, and S. TanadiniLang, "ELPHA: Dynamically deformable liver phantom for real-time motion-adaptive radiotherapy treatments," Medical Physics, vol. 46, p. 839-850, 2019

[15] S. S. Dhaliwal, T. Chettibi, A. Belarouci, G. Dherbomez, V. Coelen, and R. Merzouki, "Cooperative Brachytherapy for Prostate Cancer Under MRI Guidance," 2019 Fifth International Conference on Advances in Biomedical Engineering (ICABME), pp. 1-4, 2019.

[16] R. L. Truby, M. Wehner, A. K. Grosskopf, D. M. Vogt, S. G. Uzel, R. J. Wood, and J. A. Lewis, "Soft somatosensitive actuators via embedded 3d printing," Advanced Materials, vol. 30, no. 15, p. 1706383, 2018.

[17] G. Soter, M. Garrad, A. T. Conn, H. Hauser, and J. Rossiter, "Skinflow: A soft robotic skin based on fluidic transmission," in 2019 2nd IEEE International Conference on Soft Robotics (RoboSoft). IEEE, 2019, pp. $355-360$.

[18] J. Tapia, E. Knoop, M. Mutnỳ, M. A. Otaduy, and M. Bächer, "Makesense: Automated sensor design for proprioceptive soft robots," Soft robotics, vol. 7, no. 3, pp. 332-345, 2020.

[19] H. Yang, Y. Chen, Y. Sun, and L. Hao, "A novel pneumatic soft sensor for measuring contact force and curvature of a soft gripper," Sensors and Actuators A: Physical, vol. 266, pp. 318-327, 2017.

[20] S. Escaida Navarro, O. Goury, G. Zheng, T. M. Bieze, and C. Duriez, "Modeling novel soft mechanosensors based on air-flow measurements," IEEE Robotics and Automation Letters, vol. 4, no. 4, pp. 4338-4345, 2019.

[21] S. Escaida Navarro, S. Nagels, H. Alagi, L.-M. Faller, O. Goury, T. Morales-Bieze, H. Zangl, B. Hein, R. Ramakers, W. Deferme, G. Zheng, and C. Duriez, "A model-based sensor fusion approach for force and shape estimation in soft robotics," IEEE Robotics and Automation Letters, vol. 5, no. 4, pp. 5621-5628, 2020.

[22] V. Lagerburg, M. A. Moerland, J. J. Lagendijk, and J. J. Battermann, "Measurement of prostate rotation during insertion of needles for brachytherapy," Radiotherapy and Oncology, vol. 77, no. 3, pp. 318323, 2005.

[23] N. N. Stone, J. Roy, S. Hong, Y.-C. Lo, and R. G. Stock, "Prostate gland motion and deformation caused by needle placement during brachytherapy," Brachytherapy, vol. 1, no. 3, pp. 154-160, 2002.

[24] T. Podder, J. Sherman, D. Rubens, E. Messing, J. Strang, W.-S. Ng, and Y. Yu, "Methods for prostate stabilization during transperineal ldr brachytherapy," Physics in Medicine \& Biology, vol. 53, no. 6, p. 1563, 2008.

[25] Y. Yamada, L. Potters, M. Zaider, G. Cohen, E. Venkatraman, and M. J. Zelefsky, "Impact of intraoperative edema during transperineal permanent prostate brachytherapy on computer-optimized and preimplant planning techniques," American Journal of Clinical Oncology, vol. 26, no. 5, pp. e130-e135, 2003.

[26] C. Geuzaine and J.-F. Remacle, "Gmsh: A 3-d finite element mesh generator with built-in pre-and post-processing facilities," International Journal for Numerical Methods in Engineering, vol. 79, no. 11, pp. 1309-1331, 2009.

[27] W. C. Carson, G. J. Gerling, T. Krupski, C. Kowalik, J. Harper, and C. Moskaluk, "Material characterization of ex vivo prostate tissue via spherical indentation in the clinic." Medical Engineering \& Physics, vol. 33 3, pp. 302-9, 2011.

[28] MPX7002, -2 to +2 $\mathrm{kPa}$, on-chip signal conditioned, temperature compensated and calibrated, Freescale Semiconductor., 2021. [Online]. Available: https://www.nxp.com/docs/en/data-sheet/MPXV7002.pdf

[29] Improving ADC Resolution by Oversampling and Averaging, Silicon Laboratories Inc., 2015. [Online]. Available: https://www.silabs.com/ documents/public/application-notes/an118.pdf

[30] MPX4250D, 0 to $250 \mathrm{kPa}$, Differential, gauge pressure sensor, on-chipsignal conditioned, temperature compensated and calibrated, Freescale Semiconductor., 2017. [Online]. Available: https: //www.nxp.com/docs/en/data-sheet/MPX4250D.pdf

[31] C. Duriez, "Control of elastic soft robots based on real-time finite element method," in Robotics and Automation (ICRA), 2013 IEEE International Conference on Robotics and Automation. IEEE, 2013, pp. 3982-3987.

[32] E. Coevoet, T. Morales-Bieze, F. Largilliere, Z. Zhang, M. Thieffry, M. Sanz-Lopez, B. Carrez, D. Marchal, O. Goury, J. Dequidt et al., "Software toolkit for modeling, simulation, and control of soft robots," Advanced Robotics, vol. 31, no. 22, pp. 1208-1224, 2017. 